\title{
Detecção Automática de Anomalias Oculares Utilizando Redes Neurais Convolucionais
}

\author{
Luiza Rosa de Moura ${ }^{1}$, Alessandra Martins Coelho ${ }^{1}$, \\ Matheus de Freitas Oliveira Baffa ${ }^{1}$
}

${ }^{1}$ Departamento Acadêmico da Ciência da Computação

Instituto Federal de Educação, Ciência e Tecnologia do Sudeste de Minas Gerais

(IF Sudeste MG)

36.500-180 - Rio Pomba - MG - Brasil

\{luikta, mfreitas826\}@gmail.com, alessandra.coelho@ifsudestemg.edu.br

\begin{abstract}
Analyzing the importance of vision care, the proposed work aims to develop a method to aid the diagnosis of different anomalies that affect the external region of the eye. The Convolutional Neural Network was then used to detect visual patterns of the images in the Warsaw BioBase Disease Iris v2.1 database, which were later classified as sick and healthy. The built classifier was evaluated following the Holdout experimentation protocol, obtaining a 94 $\%$ hit rate. With this result, it is possible to conclude that the model developed has the potential to become a tool to aid diagnosis.
\end{abstract}

Resumo. Analisando a importância do cuidado com a visão, o trabalho proposto tem como objetivo desenvolver um método de auxílio ao diagnóstico de diferentes anomalias que acometem a região externa do olho. A Rede Neural Convolucional foi então utilizada para detectar padrões visuais das imagens da base de dados Warsaw BioBase Disease Iris v2.1, que posteriormente foram classificadas entre doentes e saudáveis. O classificador construído foi avaliado seguindo o protocolo de experimentação Holdout, obtendo uma taxa de acerto de 94\%. Mediante tal resultado, é possível concluir que o modelo desenvolvido tem potencial para se tornar uma ferramenta para o auxílio ao diagnóstico.

\section{Introdução}

A visão é um dos cinco sentidos do corpo humano que nos permite enxergar, perceber e interagir com o mundo. Assim, pode-se dizer que o ato de enxergar é de extrema importância para a sobrevivência humana [Borges-Osório and Robinson 2013]. Segundo [Tortora and Derrickson 2016], nos olhos estão localizados mais de 50\% dos receptores sensoriais do corpo humano, dedicando grande parte do córtex cerebral ao processamento de informação visual. Além disso, a visão humana é um complexo sistema que envolve diversas funções e diferentes órgãos e, como qualquer outro sistema do corpo, está sujeito a doenças e outras anomalias.

A oftalmologia é uma especialidade médica que tem como foco o estudo, o diagnóstico e o tratamento de doenças relacionadas com os olhos como a ambliopia, o glaucoma, a catarata, a degeneração macular, os tumores oculares, entre outros. O médico especializado nessa área é chamado de oftalmologista e possui, dentre as suas permissões, 
prescrever receitas para doenças oculares, corrigir os problemas de visão e realizar cirurgias nos segmentos clínicos e hospitalar. Os oftalmologistas também tratam de erros de refração apresentados pelos olhos, como a miopia, a hipermetropia, a presbiopia e o astigmatismo [IPMMI 2008].

Algumas doenças oculares podem gerar alterações no lado externo do olho, como as úlceras, as uveítes, o glaucoma, a ceratites (inflamações da córnea) e a iridociclites (inflamações da íris) [de Faria et al. 1997]. Além dessas, a catarata também é uma doença comum que afeta o cristalino, tornando-o opaco. A detecção dos sinais que identificam essas doenças podem requerer certa experiência do oftalmologista.

Nesta tarefa, softwares de auxílio ao diagnóstico, que funcionam de forma automática e inteligente, podem favorecer no diagnóstico mais eficaz das doenças, melhorando o prognóstico do paciente. No âmbito do desenvolvimento de sistemas de auxílio ao diagnóstico (Computer-Aided Diagnosis - CAD), sistemas inteligentes que integram técnicas de Análise e Processamento de Imagens à ferramenta de aprendizado de máquina, tem tido ampla utilização na detecção de diagnósticos complexos, como o auxílio ao diagnóstico do Edema Macular [Bhatia et al. 2020] e da Retinopatia Diabética [Gama et al. 2020].

Desta forma, o objetivo deste trabalho é desenvolver um método baseado em Visão Computacional para detectar anomalias oculares, tais como a catarata, o glaucoma, a afacia, a retinopatia diabética, as uveítes e outras condições. O método proposto integra o reconhecimento de padrões visuais a uma base de imagens de doenças oculares que afetam ou possuem sintomas característicos na região externa do olho. A principal contribuição deste trabalho é o desenvolvimento de um modelo de classificação baseado em Redes Neurais Convolucionais.

Este trabalho está dividido em cinco seções. Na Seção 2 são apresentados trabalhos relacionados ao tema proposto. A Seção 3 descreve a composição da base de dados, das classes e o pré-processamento realizado a fim de preparar as imagens para utilização nas etapas posteriores. Na Seção 4 é mostrado o desenvolvimento e a arquitetura da Rede Neural Convolucional desenvolvida. A Seção 5 demonstra os procedimentos de avaliação dos métodos, os experimentos e os resultados. Por fim, a Seção 6 conclui o presente trabalho.

\section{Trabalhos Relacionados}

O trabalho desenvolvido por [de Almeida 2018] teve como objetivo a identificação de doenças oculares. Esse objetivo foi alcançado por meio do desenvolvimento de um aplicativo para identificação da Leucocoria, que é uma anormalidade intraocular caracterizada pela presença da pupila branca e deve ser diagnosticada o mais rápido possível para evitar a cegueira. Uma das formas de identificar a doença é através de fotografias, pois, a pupila do paciente que possui a doença aparece com um aspecto branco quando utilizado o flash.

O aplicativo, desenvolvido na linguagem Java para a plataforma Android, com o uso da biblioteca OpenCV para o processamento das imagens, efetua a detecção da face, olhos e íris e apresenta o resultado da classificação da imagem para o usuário. Para realizar as análises da imagem, os autores utilizaram os algoritmos Viola-Jones, Haar Cascade e Transformada de Hough, de modo a delimitar a região do rosto e identificar os círculos da pupila e da íris. 
Para analisar o funcionamento do aplicativo foi realizada uma classificação com 53 imagens coletadas da internet, sendo 28 apenas com a região dos olhos e 25 com o rosto completo. Nos experimentos envolvendo as imagens que possuem o rosto inteiro foi obtido um total de $72 \%$ de taxa de acertos. Os autores destacaram que a aplicação possuía dificuldades em realizar a classificação de olhos claros e de imagens com determinadas posições do rosto. Também no teste realizado com as imagens que possuem apenas os olhos, o resultado não foi muito significativo, ficando em torno de $64,28 \%$ de taxa de acerto, reforçando novamente os erros observados ao testar imagens com olhos claros e determinadas posições dos olhos. Os autores concluíram que a taxa de acerto do aplicativo foi de $60,37 \%$, sendo um valor baixo relacionado aos casos de falha comentados anteriormente.

Já o trabalho de [Husemann et al. 2019] busca o desenvolvimento de uma ferramenta para auxílio ao diagnóstico de catarata em telemedicina, através de técnicas de Inteligência Artificial, para a análise e interpretação de imagens oftalmológicas. Buscaram também realizar a integração com o sistema denominado TeleOftalmoRS .

O banco de dados utilizado pelos autores era composto por imagens de exames oftalmológicos retroiluminados, com as pupilas dilatadas. O processamento de dados foi dividido em duas partes. A primeira parte teve como finalidade segmentar a área de interesse, deixando como saída a pupila e o seu contorno. A segunda parte tem como finalidade indicar a opacidade do cristalino através da imagem segmentada da pupila, indicando se o paciente possui ou não a doença.

Os resultados obtidos foram referentes à validação do algoritmo de segmentação de pupila aplicado em três grupos diferentes. O grupo 1 foi separado com 1.161 imagens, com qualidade julgada adequada para o diagnóstico. O grupo 2 com 1.544 imagens possuindo problemas na aquisição e sendo consideradas inadequadas para o exame. Já o grupo 3 foi formado com 2000 imagens de exames recentes, aguardando a análise de qualidade. Os resultados de segmentação, mostrando o percentual de acertos para cada grupo foi respectivamente $92,2 \%, 89,4 \%$ e $89,2 \%$.

Similar à proposta apresentada pelos autores correlatos supracitados, no presente trabalho é proposto um método de classificação utilizando imagens da região externa do olho. Nossa proposta, porém, aborda diferentes doenças oculares que são contempladas na construção da base de dados.

\section{Composição da Base de Dados e Pré-Processamento}

Para o desenvolvimento do trabalho foi utilizada a base de dados Warsaw BioBase Disease Iris v2.1, desenvolvida pela Universidade Tecnológica da Varsóvia (Warsaw University of Technology - WUT), na Polônia [Trokielewicz et al. 2015]. As imagens que compõem essa base de dados foram coletadas pelos criadores durante os exames de rotina oftalmológica e contemplam amostras de fotografias da região externa dos olhos de pacientes com (i) glaucoma, (ii) catarata, (iii) afacia, (iv) cegueira, (v) iridotomia, (vi) retinopatia diabética, (vii) uveítes e outras condições.

As imagens foram geradas a partir de 115 pacientes, que tiveram os seus olhos esquerdo e direito fotografados. Desta forma, a base de dados utilizada nesse trabalho é composta por 230 fotografias, referentes aos olhos esquerdo e direito de cada paciente. 
Além disso, a fim de melhorar a base de dados, 11 imagens foram removidas do teste, pois, apresentavam oclusão da íris, seja pela presença de cabelo ou dos cílios e também por apresentar dilatação farmacológica da pupila. A Figura 1 contém uma pequena amostra das imagens que compõem a base de dados.

Figura 1. Exemplos de Imagens presentes na Base de Dados.
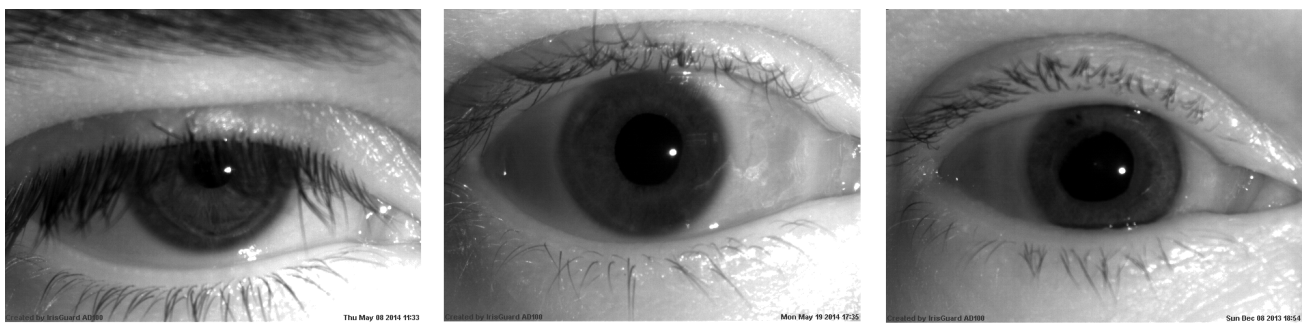

Fonte: [Trokielewicz et al. 2017].

\subsection{Segmentação Manual da Região de Interesse}

As imagens que compõe a base de dados supracitada contém informações das estruturas que compõe a região externa do olho, além de regiões não desejáveis como os cílios, a pálpebra e a sobrancelhas. Desta forma, foi realizado uma segmentação manual da região interna do olho com objetivo de limitar a região o qual o algoritmo de classificação irá detectar padrões. Assim, pode-se esperar a aquisição de padrões melhores referentes a detecção das anomalias oculares. A Figura 2 contém um exemplo de antes e depois do recorte em uma das imagens que compõem a base de dados.

Figura 2. Imagem antes e depois do recorte.

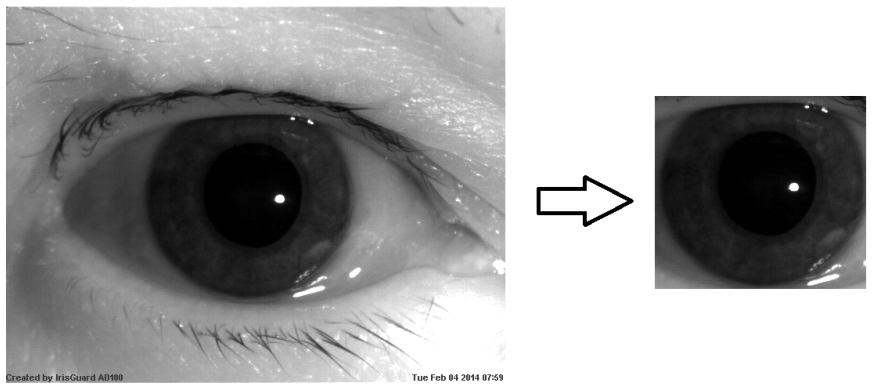

Fonte: autor.

\subsection{Melhorando de Base de Dados}

A fim de aumentar o número de amostras da base de dados, uma etapa de melhorando (data augumentation) foi incluída. Nesta etapa, as imagens que compõem a base foram submetidas a dez operações de processamento de imagens como a rotação, a inversão e o recorte. Ao final desse processo, a base de dados resultante foi composta por 2.190 imagens, sendo elas 1.095 provenientes de pacientes saudáveis e 1.095 imagens provenientes de pacientes com alguma anomalia. 


\section{Desenvolvimento e Configuração da Rede Neural Convolucional}

O método de classificação proposto, foi desenvolvimento baseado nas Redes Neurais convolucionais (Convolutional Neural Network - CNN), e inspirada na arquitetura tradicional de LeNet-5. A CNN então foi composta por três camadas de convolução de duas dimensões (2D Conv. Layer), extraindo 32, 64 e 128 filtros, respectivamente, de tamanho 3x3, seguidos por uma operação de MaxPooling de 2x2, a qual reduz a dimensão da imagem pela metade antes de propagá-la para as próximas camadas internas da rede.

Além das camadas responsáveis pela detecção de padrão, uma camada de achatamento (flatten) foi utilizada com objetivo de converter os padrões detectados no formato matricial (feature maps), para um vetor descritor. Este vetor é posteriormente enviado a uma Rede Neural Totalmente Conectada (Fully-Connected Neural Network), composta por duas camadas densas (dense layers) com 1024 neurônios cada, responsáveis pela detecção de padrões no vetor descritor da imagem. Esta camada utiliza neurônios com a função de ativação Rectifier Linear Unit (ReLU). Agregado a esta camada, a operação de dropout é realizado com objetivo de desligar aproximadamente $20 \%$ dos neurônios, auxiliando na atribuição de um conhecimento mais generalizado sobre a base de dados, possibilitando uma classificação mais generalizada e sem overfitting. Além disso, o tamanho do batch utilizado pelo algoritmo foi tamanho 16. A Figura 3 contém um modelo ilustrando a arquitetura desenvolvida de Rede Neural Convolucional.

Figura 3. Arquitetura da Rede Neural Convolucional desenvolvida.

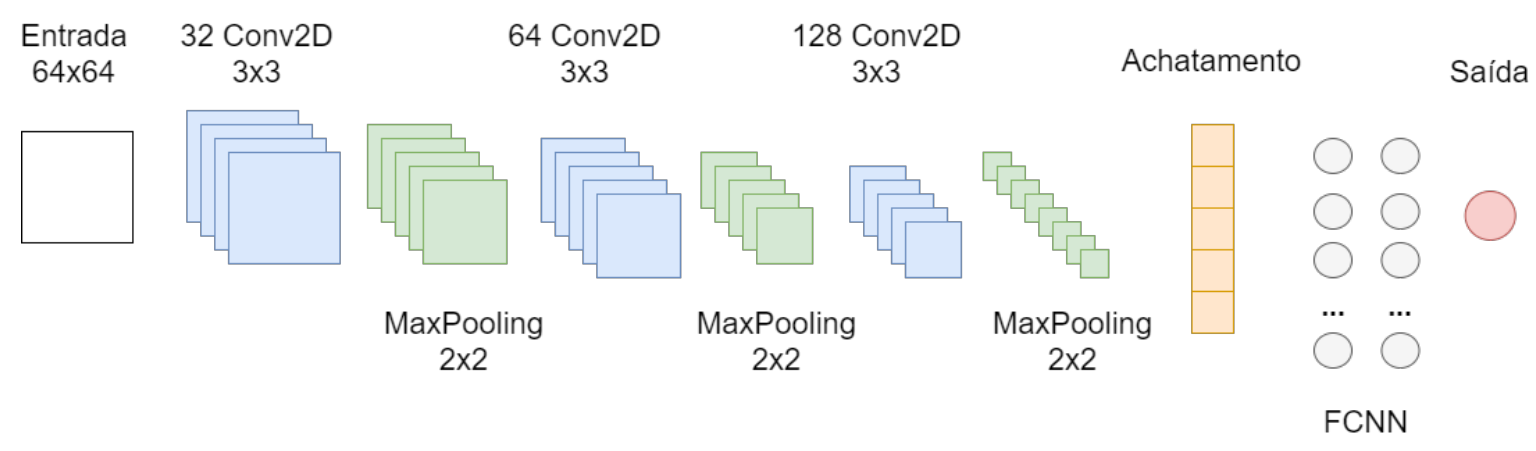

Fonte: autor.

\section{Experimentos e Resultados}

A linguagem Python na versão 3.7, em conjunto com as bibliotecas OpenCV 4.0 e o Tensorflow 2.3 foram utilizados para a implementação da proposta. Para o treinamento da rede neural foi utilizado um computador com processador Ryzen 7 1700, 16 GB de memória RAM DDR4 e uma GPU NVIDIA GTX 1060, rodando no sistema operacional Windows 10 Pro.

Os experimentos foram conduzidos seguindo o protocolo de experimentação $\mathrm{Hol}$ dout. Este protocolo dita que parte da base de dados deve ser utilizada para treinamento e parte para a validação, de forma que as imagens não se repitam por entre as sub-bases de dados. Desta forma, neste trabalho consideramos $70 \%$ da base de dados para treinamento e $30 \%$ para validação.

O experimento avaliou a capacidade da arquitetura proposta em detectar padrões na base de dados utilizada. Para isso, foram calculadas quatro métricas de avaliação, 
Tabela 1. Resultados obtidos pelo experimento Holdout.

\begin{tabular}{cl}
\hline Métrica & Resultado \\
\hline Acurácia & $94,44 \%$ \\
Precisão & $94,39 \%$ \\
Revocação & $96,19 \%$ \\
AUC & $98,06 \%$ \\
\hline
\end{tabular}

sendo elas a acurácia, a precisão, a revocação e a área abaixo da curva ROC (Area Under the Curve - AUC). Os resultados obtidos pelo método, pode ser observado na Tabela 1.

Figura 4. Gráfico de acurácia por época.

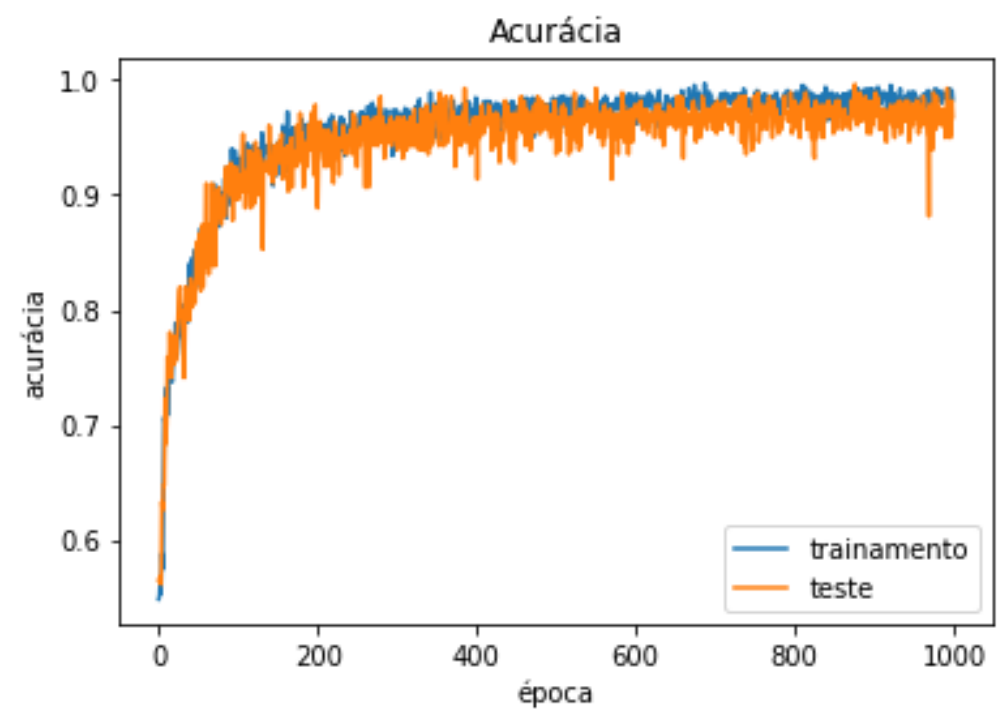

Fonte: autor.

Analisando os resultados, é seguro afirmar que o algoritmo conseguiu distinguir ambas as classes propostas no problema de classificação binária, doente e saudável, não tendendo para nenhuma classe específica. Além disso, os valores de acurácia do teste e da validação ao longo do treinamento, se mantiveram próximas, indicando que não houve também, sobreajuste do modelo de classificação à base de dados uma vez que, a validação em imagens fora do escopo do treinamento foi realizada de forma satisfatória. Essa curva pode ser observada no gráfico acurácia por época, representado na Figura 4.

\section{Conclusão}

A visão é uma das mais importantes fontes sensoriais do corpo humano. Esta é responsável pela nossa percepção e interação com o mundo. Neste trabalho, foi desenvolvido um método de detecção de anomalias da região externa do olho, utilizando Redes Neurais Convolucionais. O método proposto atingiu uma acurácia satisfatória, de aproximadamente $94 \%$. É importante salientar que a detecção correta destas anomalias, mesmo que em pequenas escalas, pode promover um melhor prognóstico ao paciente, evitando a cegueira e lhe proporcionando melhor qualidade de vida. Espera-se que em trabalhos futuros, uma análise multiclasse da base de dados seja realizada, classificando não so- 
mente se uma determinada imagem é proveniente de um paciente doente ou saudável, mas também indicando qual a anomalia e, qual região do olho o padrão foi detectado.

\section{Agradecimentos}

Os autores agradecem ao IF SudesteMG e ao Programa de Educação Tutorial (PET Conexões de Saberes Ciência da Computação) pelo apoio técnico e financeiro para o desenvolvimento do projeto.

\section{Referências}

Bhatia, K. K., Graham, M. S., Terry, L., Wood, A., Tranos, P., Trikha, S., and Jaccard, N. (2020). Disease classification of macular optical coherence tomography scans using deep learning software: validation on independent, multicenter data. Retina, 40(8):1549-1557.

Borges-Osório, M. R. and Robinson, W. M. (2013). Genética Humana 3ed. Artmed Editora.

de Almeida, R. S. (2018). Iris: Aplicativo para identificação de leucocoria. Disponível em: http://dsc.inf.furb.br/arquivos/tccs/monografias/2018 2_rafael-sabel-dealmeida_monografia.pdf. Acesso em: 08 mar. 2021.

de Faria, S. J. et al. (1997). Doenças oculares externas. Medicina (Ribeirao Preto), 30(1):52-55.

Gama, Í., Coelho, A., and Baffa, M. (2020). Fundus eye images classification for diabetic retinopathy detection using very deep convolutional neural network. In Anais do XVI Workshop de Visão Computacional, pages 24-29. SBC.

Husemann, R., Negreiros, M., Tomaggi, H., Araujo, A. L., and Roesler, V. (2019). Desenvolvimento de uma ferramenta para auxílio ao diagnóstico de catarata em telemedicina. In Anais Estendidos do XXV Simpósio Brasileiro de Sistemas Multimídia e Web, pages 155-158. SBC.

IPMMI, H. e. M. M. K. B. (2008). Oftalmologia. Disponível em: http://www.hospitalmarieta.com.br/. Acesso em: 08 mar. 2021.

Tortora, G. J. and Derrickson, B. (2016). Corpo Humano-: Fundamentos de Anatomia e Fisiologia. Artmed Editora.

Trokielewicz, M., Czajka, A., and Maciejewicz, P. (2015). Database of iris images acquired in the presence of ocular pathologies and assessment of iris recognition reliability for disease-affected eyes. In 2015 IEEE 2nd International Conference on Cybernetics $(C Y B C O N F)$, pages 495-500. IEEE.

Trokielewicz, M., Czajka, A., and Maciejewicz, P. (2017). Implications of ocular pathologies for iris recognition reliability. Image and Vision Computing, 58:158-167. 